\title{
Surgery for Benign Colorectal Disease Improves Patient-reported Quality of Life
}

R. Maniar ${ }^{1}$, G. Liư ${ }^{2}$, J. Sutherland ${ }^{2}$, C. Brown ${ }^{1}$, M.Ravall ${ }^{1}$, T. Phang ${ }^{1}$, and A. Karimuddin ${ }^{1}$ ${ }^{1}$ Department of Surgery, ${ }^{2}$ School of Population and Public Health, University of British Columbia, Vancouver, Canada

\section{Introduction}

- Patient-reported outcomes (PRO) on health status and quality of life (QOL) can be used to assess surgical intervention, yet there is limited data on PRO for patients with benign colorectal disease

\section{Purpose}

- To assess if PRO on health status improved after elective major abdominal surgery for benign colorectal disease

\section{Methods}

- Patients with Crohn's disease, Ulcerative colitis or diverticular disease undergoing elective major abdominal surgery were surveyed on PRO from 2012 to 2016

- Procedures included small or large bowel resection, or pelvic pouch procedure

- Health status PRO were collected after placement on the surgical wait list and 6 months post-operatively

EQ-5D index and visual analog scale (VAS)

- PEG pain scale and PHQ-9 depression scoring tool

\begin{tabular}{|c|c|}
\hline $\begin{array}{c}\text { Placement on the surgical } \\
\text { waitlist }\end{array}$ & $\longrightarrow \begin{array}{c}\text { Complete pre-operative health } \\
\text { surveys }\end{array}$ \\
\hline 6 months post-operative & $\begin{array}{c}\text { Complete post-operative } \\
\text { health surveys }\end{array}$ \\
\hline
\end{tabular}

\section{Results}

- 57 patients completed both pre- and post-operative surveys

- $93 \%$ of patients had no other comorbidities (Charleston comorbidity index $=0$ )

Patient Demographics

\begin{tabular}{|l|l|l|r|}
\hline $\begin{array}{l}\text { Characteristic } \\
\text { Diagnosis }\end{array}$ & Percent (N) & Characteristic & Percent (N) \\
\hline $\begin{array}{c}\text { Crohn's } \\
\text { UC }\end{array}$ & $28.1 \%(16)$ & Procedure & \\
\hline Diverticulitis & $52.6 \%(30)$ & Segmental colectomy & $22.8 \%(13)$ \\
\hline Approach & & Anterior resection & $47.4 \%(27)$ \\
\hline MIS & $61.4 \%(35)$ & Proctocolectomy/APR & $10.5 \%(6)$ \\
\hline Open & $38.6 \%(22)$ & Pelvic Pouch & $10.5 \%(6)$ \\
\hline
\end{tabular}

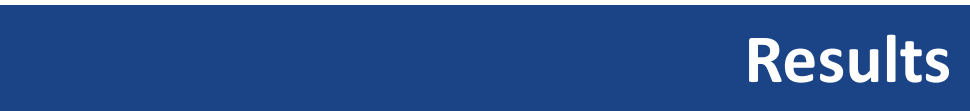

Health Status PRO for benign disease

\begin{tabular}{|l|c|c|c|}
$\begin{array}{l}\text { Survey } \\
\text { Instrument }\end{array}$ & $\begin{array}{c}\text { Pre-operative score } \\
\text { (mean } \pm \text { standard } \\
\text { deviation) }\end{array}$ & $\begin{array}{c}\text { Post-operative score } \\
\text { (mean } \pm \text { standard } \\
\text { deviation) }\end{array}$ & -value \\
\hline EQ-5D (/1.0) & $0.78 \pm 0.19$ & $0.85 \pm 0.17$ & 0.004 \\
\hline VAS (/100) & $66.9 \pm 19.7$ & $76.2 \pm 18.0$ & 0.001 \\
\hline PEG (/10) & $2.9 \pm 3.1$ & $2.0 \pm 2.4$ & 0.020 \\
\hline PHQ-9 (/27) & $5.3 \pm 5.4$ & $3.7 \pm 4.4$ & 0.016
\end{tabular}

\begin{tabular}{|c|c|c|c|}
\hline $\begin{array}{l}\text { Survey } \\
\text { Instrument }\end{array}$ & Pre-operative score & Post-operative score & $p$-value \\
\hline EQ-5D (/1.0) & & & \\
\hline Diverticulitis & $0.79 \pm 0.17$ & $0.88 \pm 0.16$ & 0.01 \\
\hline Crohn's & $0.69 \pm 0.23$ & $0.81 \pm 0.21$ & 0.05 \\
\hline UC & $0.89 \pm 0.10$ & $0.85 \pm 0.10$ & 0.71 \\
\hline VAS (/100) & & & \\
\hline Diverticulitis & $67.7 \pm 14.9$ & $78.7 \pm 15.6$ & 0.005 \\
\hline Crohn's & $56.3 \pm 23.3$ & $68.3 \pm 22.9$ & 0.05 \\
\hline UC & $80.4 \pm 16.6$ & $81.3 \pm 13.7$ & 0.88 \\
\hline PEG (/10) & & & \\
\hline Diverticulitis & $2.7 \pm 2.7$ & $1.6 \pm 2.1$ & 0.05 \\
\hline Crohn's & $4.3 \pm 3.6$ & $2.8 \pm 3.2$ & 0.04 \\
\hline UC & $1.2 \pm 2.4$ & $1.8 \pm 1.3$ & 0.59 \\
\hline PHQ-9 (/27) & & & \\
\hline Diverticulitis & $3.8 \pm 3.9$ & $2.8 \pm 3.2$ & 0.19 \\
\hline Crohn's & $9.3 \pm 7.2$ & $6.7 \pm 6.4$ & 0.05 \\
\hline UC & $3.4 \pm 2.7$ & $2.7 \pm 1.6$ & 0.49 \\
\hline
\end{tabular}

\section{Conclusion}

- Surgical intervention for benign colorectal disease improves patientreported health status and QOL

- Improvements in PRO for Crohn's disease and diverticulitis may be due to pre-operative symptom burden, and these patients may benefit from pain and depression optimization before surgery

- Surgery for ulcerative colitis may not result in significant improvements in patient-reported health status as measured by the EQ-5D, PEG and PHQ9 tools

- Patients with benign colorectal disease can be counselled their health status will likely remain stable or significantly improve following elective abdominal surgery - Further study is needed to determine which perioperative factors other than disease process influence patients' QOL and health status 